\title{
Review Paper on Crop Area Estimation Using SAR Remote Sensing Data
}

\author{
Priyanka Shewalkar ${ }^{1}$, Anand Khobragade ${ }^{2}$, Prof. Kapil Jajulwar ${ }^{3}$ \\ ${ }^{I}$ Research scholar, G.H.Raisoni College of Engineering, Nagpur \\ ${ }^{2}$ Resources Scientist, Maharashtra Remote Sensing Applications Centre, Nagpur \\ ${ }^{3}$ Asst. Professor, G.H.Raisoni College of Engineering, Nagpur
}

\begin{abstract}
Crop detection is a critical and evaluative first step towards almost agricultural monitoring systems. The ability to identify crop type makes it possible to estimate the agricultural area allocated under crop and hence compute relevant statistics providing essential information for crop control of area-based subsidies The capabilities of SAR for discriminating crop type have been previously explored. The main aim is to brief the mechanism of crop acreage estimation and area monitoring using SAR data, Envisat-ASAR, or polarimetric SAR with multi-temporal and multi polarized datasets and review the research progress on crop estimation and area monitoring using microwave remote sensing.
\end{abstract}

Keywords: crop estimation; area monitoring; SAR; remote sensing.

\section{Introduction}

Identification of crop types is the first step of crop monitoring system and crop yield forecasting. The traditional ground survey methods is difficult to acquire annual crop information due to the less economic efficiency and some features of agricultural production, for e.g., the large coverage, the strong seasonal, strong spatial heterogeneity, using remote sensing technology is feasible and effective way to solve this problem. The achievements are remarkable, since remote sensing was used to crop identification and area extraction, the technology and theory have been in continuous improvement.

Microwave remote sensing has the following characteristics, Firstly, the most obvious difference from optical remote sensing is its capability to penetrate clouds and to some extent rain. Secondly, microwave is independent of the sun as a source of illumination, so it has the ability of all-day working. Thirdly, microwave is able to penetrate deeper into vegetation than optical waves can, so, when it is used to monitor vegetation, not only can acquire the surface information of the vegetation, but also can have some reflection of the vegetation leaves, stems, branches, stem and other structure information under the surface of vegetation. Fourthly, the received signal by the microwave sensor is mainly affected by the structural characteristics and dielectric properties of the surface features, so this information can reflect the surface features of the objects which may vary from the optical remote sensing reflect.

SAR data with one polarization (single date) gives relatively low classification accuracy (around $69 \%$ ). Hence, the first objective to study the multipolarization data in improving crop mapping accuracy.

\section{Research Trends}

The temporal variation of backscattering coefficient $\left(\sigma^{\circ}\right)$ of rice is larger than other crops by analyzing crops' backscattering characteristics in X-band SAR image and firstly proposed the possibility of monitoring and calculating rice production using SAR image. Many researchers proposed comprehensive utilization of multi-temporal data. $\mathrm{HH}$ with $\mathrm{HH} / \mathrm{HV}$ is the best polarization combination for rice identification under the adoption of linear polarization combination. ASAR data with dual-polarization measurement capability is also used for rice monitoring. It was observed that backscattering coefficient of rice is more dissimilar in linear polarizations (HH, HV, VH and VV), while less in circular polarizations (RR, LR and LL). The accuracy of rice identification is up to $90 \%$, greater than RADARSAT-2, mainly because airborne SAR data has higher resolution. After the emergence of polarimetric SAR satellite, the RADARSAT-2, there will be great potential to fully explore the polarimetric SAR data to monitor rice plantation acreage and estimate the production of rice [1]. Performing the classification of rice using multi-temporal Radarsat-1 data, using the changes of different backscattering coefficient in different crop growth stage to distinguish rice and its symbiotic vegetation, the results achieve a more high accuracy and meet the application requirements. Comparing to single-band and single-phase data, the multi-polarization and multi-temporal data have shown the markedly merits in crop classification and area monitoring, like the data EnviSat/ASAR, ALOS/PALSAR, TerraSAR-X, Radarsat-2 data all can provide multi-polarization mode. The combination of multi-incidence angle, multitemporal and multi-polarization data is one of applications trend of radar data in crop classification and area monitoring [2]. Based on the rice survey project of background data proposes the crop 
identification method on two- phase classification in large scale (2PCM), and a process of running technique. This paper includes the first-phase classification, sampling, the second-phase classification and extrapolation. MODIS image were used as the datasets. MODIS images with large coverage, high temporal resolution and low cost even free are taken as the first-phase identification data source for the classification identification of RS images. Secondly, using the scatter gram as a sampled population, combining with the group sampling method, an object of second- phased, classification identification is obtained for making the use of STOP images to execute second- order on the sample region. Last, the extrapolation method is applied according to target in sampling areas. [3].

Another image dataset is ASAR, which has the features of multi-mode, multi-polarization, large swath and multi-incident angles. The ASAR Data Pre-processing for the research includes 4 steps: radiometric calibration, mutual calibration among multi-temporal images, the multi-temporal wave filter of speckle noise and geometry correction. Total accuracy of $82.64 \%$ for wheat/non-wheat classification was obtained in this paper [4]. Worldview2 imagery is primarily used and compared with QuickBird imagery. Supplementary value of the new 4 bands (coastal, yellow, red edge, and NIR2) in WorldView-2 imagery is discussed [5].

Another paper in which MODIS data as examples, the extracted spectral characteristics are analyzed using statistical method and dynamic changes of temporal series of indices including NDVI, EVI, MSAVI and NDWI are studied and an AFSM (Adaptive Feature Selection Model) is developed. MODIS data is divided into four phases: the RS image preprocessing, feature extracting, feature selection with AFSM and crop identification [6].

\section{Results \& Discussion}

In recent years, crop identification and area monitoring using SAR data is being given more and more attention. To some extent, SAR data has the advantage that cannot influenced by the rain and cloud weather and can penetrate crop in a certain range and the merits make a great contribution to the prevailing trend. The combination of multi-incidence angle, multi-temporal and multi-polarization data is one of applications trend of radar data in crop detection and area monitoring in recent years. With the increased choice of radar data sources, combination of different dimensional information like incidence angle, phase and polarization certainly improve the accuracy of crop identification, but, at the same time, many choices can also cause data redundancy.

After a detailed literature survey it is found that authors have experimented on various datasets such as SAR image, Envisat-ASAR image, and RADARSAT-2 image, with the combination of multi-incidence angle, multitemporal and multi-polarization data.

\section{References}

[1]. Kun Li, Yun Shao, Fengli Zhang, "Paddy Rice identification using polarimetric SAR data in Southern China", IEEE, 2010.

[2]. DING Yaping, CHEN Zhongxin, "A review of crop identification and area monitoring based on SAR image", China, 2012 IEEE.

[3]. Qiong An, Wanlin Gao, Lina Yu, Jianjia Wu, Bangjie Yang, Wei Liu, "Research on Crop Identification Method Based on TwoPhase Classification Using Remote Sensing in Large Scale", China, 2011 IEEE

[4]. Bai Lina, Wang Bengyu, Tian Xin, Lu Ying, Yang Yongtian, "Winter Wheat Identification Using Multi-Temporal Envisat Asar Data --A Case Study At Tongzhou District, Beijing”, IGARSS, 2012

[5]. Kadim Tasdemir, Selcuk Reis, "Land Cover Identification for Finding Hazelnut Fields Using Worldview-2 Imagery", IEEE 2011

[6]. Qiong An, Wanlin Gao, Bangjie Yang, Jianjia Wu, Lina Yu, Zili Liu, "Research on Feature Selection Method Oriented to Crop Identification Using Remote Sensing Image Classification", Sixth International Conference on Fuzzy Systems and Knowledge Discovery, IEEE, 2009 